\title{
De-risking flight trials using airwake simulations
}

\author{
C M Ward* MEng PhD CEng MIMechE \\ * Frazer-Nash Consultancy, UK, \\ * Corresponding author. Email: c.ward@fnc.co.uk
}

\begin{abstract}
Synopsis
Air operations around naval vessels are inherently challenging and a major contributor to this is the turbulent airflow around the vessels, colloquially known as the airwake. To manage the risks associated with these unsteady airflows and to help define safe operating limits for the ship and the aircraft, the Royal Navy undertakes First of Class Flight Trials (FOCFTs). However, these trials inherently carry their own risks as well as being costly and time consuming. This paper discusses how Computational Fluid Dynamics (CFD) simulations have been used to de-risk flight trials and operations on the Queen Elizabeth Class (QEC) carriers. The simulations are shown to be in excellent agreement with full-scale LiDAR and anemometer measurements, which provides the requisite confidence to use them as a basis for de-risking.

To de-risk the rotary wing FOCFTs, the turbulence approach parameter was defined as a proxy for pilot workload. It is shown that this parameter can be used to identify the wind conditions that are likely to be the most difficult for pilots, and to advise on changes to the approach paths that would reduce pilot workload. Test pilots were briefed with this airwake information prior to the FOCFTs, and the flow features identified in the CFD were found to be consistent with the pilots' experiences. In the future this analysis could be used to reduce the time and cost associated with flight trials, manage through-life risks, and assess the impact of design decisions on the airwake during ship design. The work has also been used to de-risk F-35 trials and operations. In particular, the findings show that it may be possible to extend the operating envelope of the aircraft using a novel real-time system to predict airwake turbulence. In addition, CFD simulations were used to de-risk ondeck operations by ensuring that aircraft are within their exposure limits when tied-down. This information was used by the FOCFTs teams during rotary wing trials.
\end{abstract}

Keywords: Airwake; De-risking; Flight trials; Simulation; CFD; Ship-air interface

\section{Introduction}

The Queen Elizabeth Class (QEC) carriers are the largest aircraft carriers ever built for the Royal Navy. Their primary purpose is to act as a base for aircraft and, therefore, it is vital that aircraft are able to take-off and land safely on the deck in a wide range on environmental conditions. Air operations around any naval ship are inherently challenging. A major contributor to this is the turbulent airflow around the ship, colloquially known as the aiwake, which can make it difficult for pilots to operate safely. To mitigate the risks associated with shipair interface activities, the Royal Navy currently undertakes First of Class Flight Trials (FOCFTs) to define suitable safe operating envelopes for the ship and aircraft. However, these trials inherently pose a risk to both the assets (aircraft and ship) and personnel, as well as being time consuming and costly.

One method of reducing the risks associated with FOCFTs is to use flight simulators to train pilots in a low risk environment, and a key component of the flight simulators is the airwake around the vessel. As part of this programme of work, Computational Fluid Dynamics (CFD) modelling was used to generate airwake datasets for flight simulators. However, the CFD modelling techniques required for this are not new and have been widely studied [Polsky, 2016] and so the details of this are not discussed here. Instead, this paper focuses on the other novel ways in which the CFD models have been used to de-risk flight trials and operations, and how they could be used in the future to reduce time and costs, manage through-life risk, and expand aircraft operating envelopes.

Section 2 of this paper provides an overview of the CFD simulations undertaken as part of this work and Section 3 then discusses work that has been undertaken to provide confidence in these results, including comparisons to LiDAR and anemometer measurements. Sections 4, 5 and 6 discuss how the simulations have been used to derisk rotary wing trials and operations, F-35 trials and operations, and on-deck operations respectively. 


\section{Overview of the CFD simulations}

Two types of CFD simulations were used in this work, steady state simulations and transient simulations. Steady state simulations were used to provide high-level assessments of the airwake, to identify flow features of interest to pilots and de-risk on-deck operations. Transient simulations were used to provide a more detailed understanding around unsteady flow features of the airwake, as well as generating the datasets for use in the flight simulators.

All of the simulations were undertaken using the commercial software package Fluent v17. For the steady simulations, a Reynolds-Averaged Navier Stokes (RANS) approach was implemented with the k- $\omega$ SST turbulence model. The transient simulations were computed using a Large Eddy Simulation (LES) approach. Both sets of simulations used the same mesh, which comprised approximately 70 million cells. Best practice checks were undertaken to ensure numerical accuracy and suitable solution convergence, including: ensuring the mesh was suitably refined to explicitly capture at least $80 \%$ of the turbulent kinetic energy [Pope, 2000]; checking sub-time step convergence; and ensuring the domain was washed through before capturing any transient airwake data.

\section{Validation of modelling results}

In order for the CFD modelling to be used in de-risking trials and operations, it was critical that there was sufficient confidence in the results. To provide this confidence, the results of the simulations have been compared to full-scale measurements. This section discusses the resulting validation of the CFD modelling.

\subsection{Validation of the flow behind the vessel}

The University of Exeter has conducted an independent comparison of the results of the transient CFD models to full-scale experimental LiDAR (Light Detection and Ranging) measurements of the airflow [Christmas, 2018]. The details of this assessment are not covered here; however, an example of the comparison is provided in Figure 1. The experimental results shown in this figure were obtained from a LiDAR mounted on the deck, facing aft along the F-35 Shipborne Rolling Vertical Landing (SRVL) approach path as indicated in the figure. These images show the flow velocity measured along a LiDAR beam. A vertical slice through these plots gives the flow velocities along the LiDAR beam at an instance in time, and a horizontal slice shows how the velocity at a point in space varies with time. Therefore, the diagonal structures that can be seen are flow features convecting away from the ship along the LiDAR beam.

The images show that the results from the CFD simulations agree very well with the LiDAR measurements, both in terms of the magnitude and extent of the wake and the scale of the flow features. This provides a high degree of confidence that the CFD is correctly capturing the vortices shed from the ship and their convection downstream along with the effects of atmospheric turbulence. 

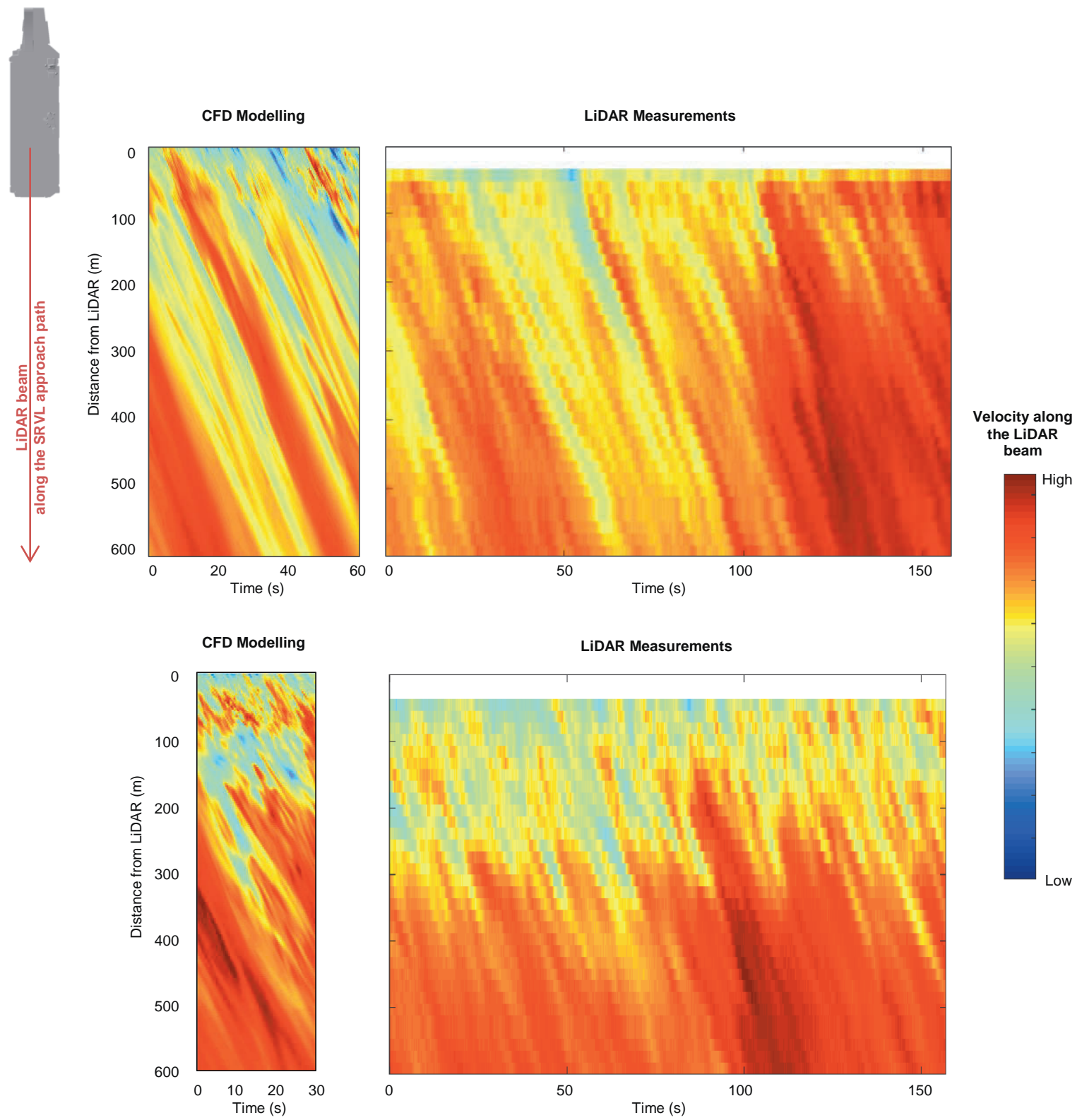

Figure 1: Comparison of flow velocity aft of the vessel predicted by the CFD (left) and measured by LiDAR (right) [Christmas, 2018] for Straight Ahead (top) and Green $10^{\circ}$ (bottom) winds.

\subsection{Validation of flow over the deck}

The results of the transient CFD simulations were also compared to anemometer measurements taken over the deck. Figure 2, which has been taken from the comparison report [Christmas, 2018], shows the local wind speed and direction measured by an on-deck anemometer, relative to the freestream conditions (as measured by the LiDAR). The figure also shows the relative wind speed and direction predicted by the CFD at the anemometer location. The results show very good agreement between the CFD and the anemometer, which demonstrates that the CFD is correctly capturing the details of the local flow around the vessel.

The close agreement between the LiDAR, CFD and the anemometer also shows that CFD modelling can be used to improve the accuracy of the anemometer system by providing a calibration to correct for local flow effects. This improvement in accuracy would in turn reduce the risks associated with uncertainty in the wind conditions when conducting ship-air operations. 


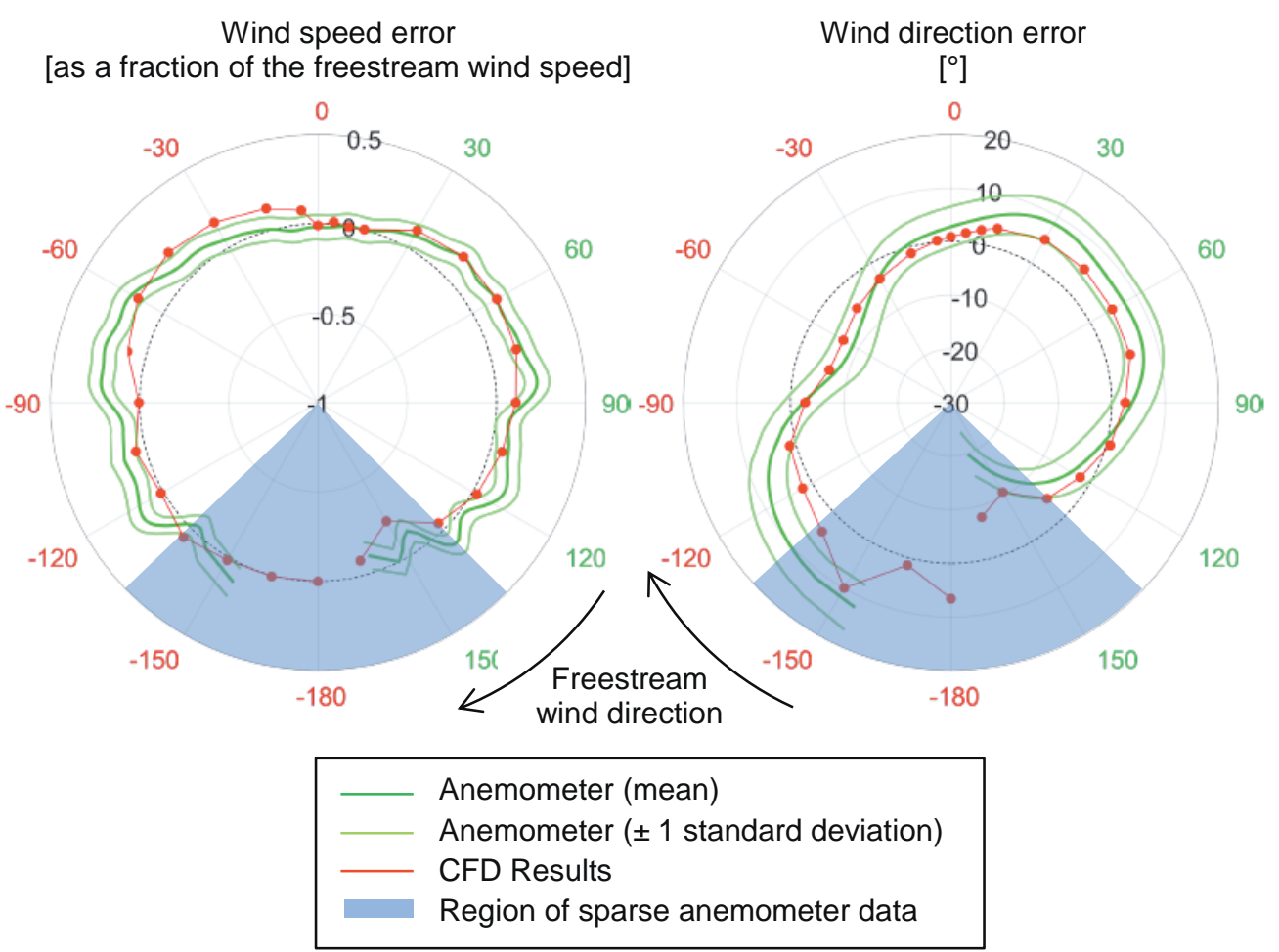

Figure 2: Local wind speed (left) and direction (right) measured by the anemometers (green) and predicted by CFD (red) relative to the freestream conditions [Christmas, 2018].

\section{De-risking rotary wing trials and operations}

This section summarises the assessments undertaken to de-risk the FOCFTs for Merlin and Chinook. QinetiQ FOCFTs teams and test pilots were briefed with the results of these assessments prior to the trials.

\subsection{General airwake observations}

In order to understand the airwake around the ship, the flow was assessed on a plane above the flight deck. Typical images from this assessment are presented in Figure 3. This figure shows contours of turbulent kinetic energy and mean vertical velocity around the QEC carrier in Ahead, Red and Green winds. The dashed line on these images is a representative approach path to a landing spot on the deck. For the approach shown:

- In Ahead winds, both the turbulent kinetic energy and the mean vertical velocities around the vessel are relatively small. Consequently, these winds are expected to be relatively benign compared to Red or Green winds at the same wind speed.

- In Green winds, the wakes from the islands are the dominant flow features of interest. These wakes wash over the approach path and are likely to make this a turbulent approach compared to other wind directions.

- In Red winds, the turbulence levels along the approach are much lower because the approach is largely upstream of the wake. However, the turbulence is likely to increase when transitioning over the landing spot due to a large vortex which rolls up off the port side of the vessel, as shown in Figure 4. This vortex also causes a large change in vertical velocity, so when transitioning across the deck the aircraft will move from a region of strong up-draft to a region of strong down-draft. This will be notable to pilots as an increase in the decent rate. 

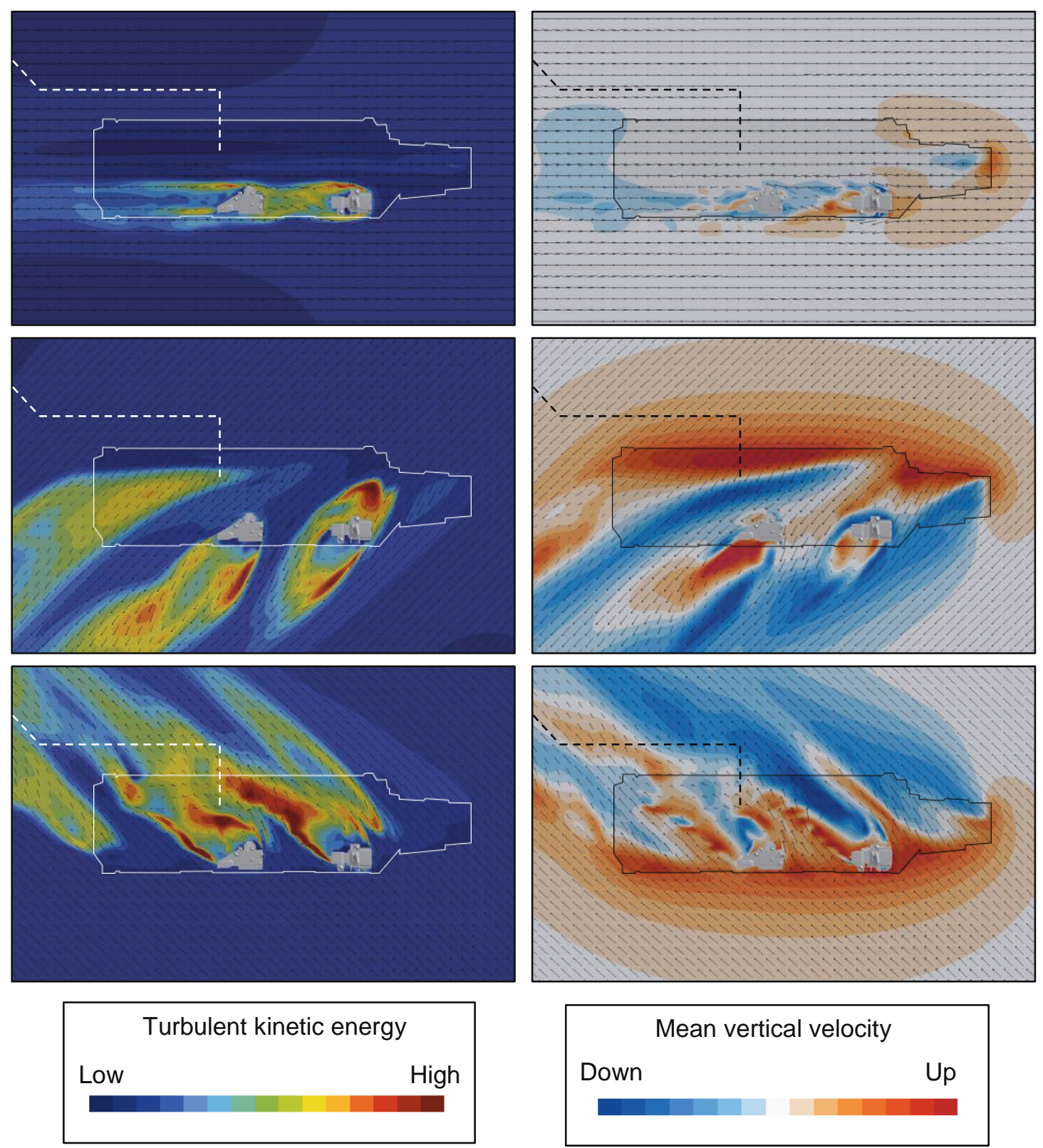

Figure 3: Contours of turbulent kinetic energy (left) and mean vertical velocity (right) in Ahead (top), Green (middle) and Red (bottom) winds. Representative approach path shown as a dashed line. 


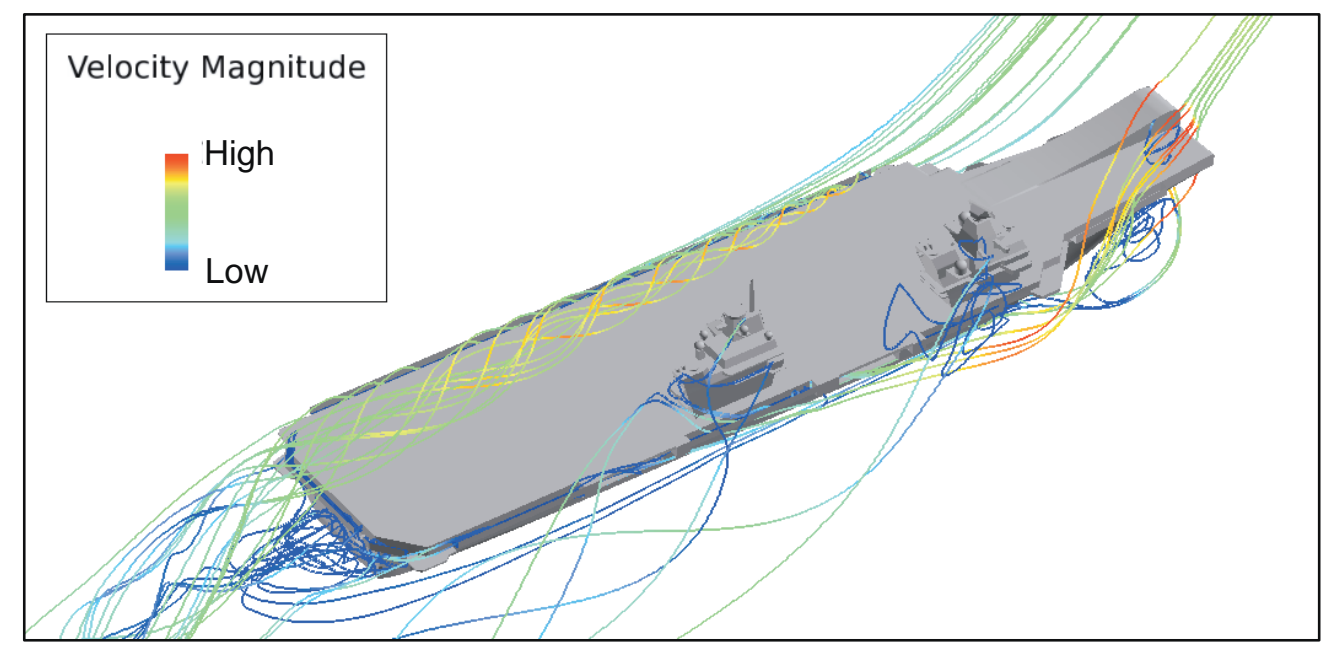

Figure 4: Streamlines showing the vortex rolling up along the port side of the vessel in red winds.

\subsection{Approach turbulence assessment}

The ability to quantify approach 'difficulty' is useful for decision making purposes. In this work the turbulent kinetic energy of the flow, $k$, has been used as a basis for quantitative assessment. Higher values of turbulent kinetic energy are expected to result in stronger buffeting of the aircraft, and therefore a higher pilot workload. It should be noted that other factors contributing to the overall pilot workload including ship motion, visibility and on-deck activity, and are not considered in this analysis.

Figure 5 shows the variation of the average turbulent kinetic energy over the rotor disk area, $A_{\text {rotor }}$, during a typical approach. As shown in the figure, the variations in turbulence can be linked to particular flow features, and the parts of the approach likely to be the most difficult for pilots can be identified. In the example shown, the final hover before descending to the landing spot will be subject to the greatest turbulence due to its position in the middle of the wake from the forward island.

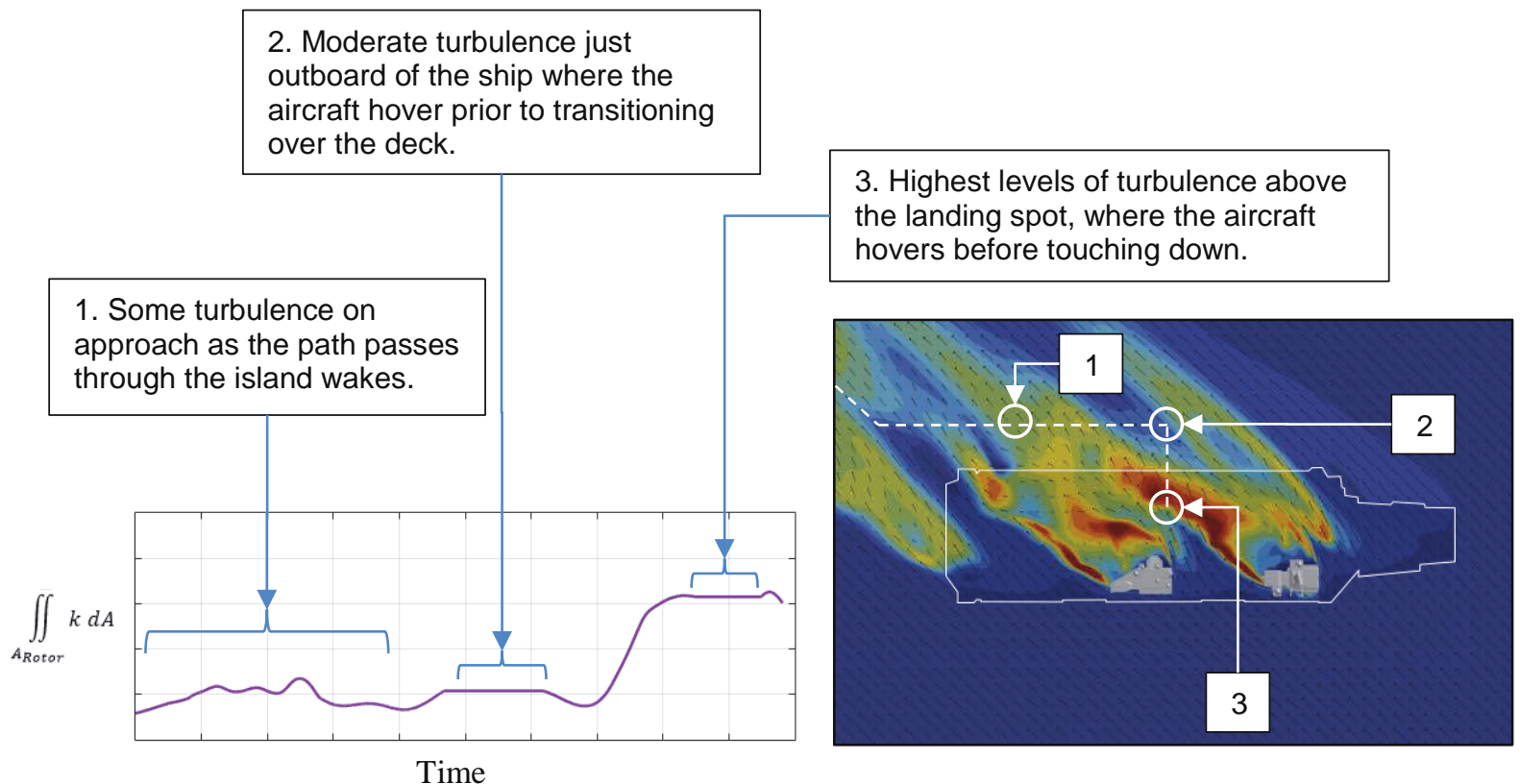

Figure 5: Variation of turbulence during a typical approach in Green wind (left) and the corresponding contours of turbulent kinetic energy (right).

To capture the approach 'difficulty' in a single value, the approach turbulence parameter, $P$, has been defined as the integral of the rotor-averaged turbulent kinetic energy over the duration of the approach, $T$. 


$$
P=\int_{0}^{T} \iint_{A_{\text {Rotor }}} k d A d t
$$

The approach turbulence parameter provides a measure of how turbulent an approach will be for a given wind condition. Low values indicate that the pilots will not experience much turbulence, while high values indicate the approach involves sustained periods of time in highly turbulent flow.

Figure 6 shows how the approach turbulence parameter varies with wind direction for the approach to six landing spots. This assessment agrees with the qualitative assessment described in Section 4.1: Ahead winds result in the lowest levels of turbulence; Red winds result in moderate levels of turbulence; and Green winds result in the strongest levels of turbulence. A post-trials debrief with the FOCFTs teams indicated that these conclusions are consistent with the pilots' experience.

In the future, this quantitative assessment could also be used to inform and de-risk flight trials in several other ways:

1. To help plan flight trials programmes (e.g. to help prioritise which wind directions should be tested by pilots). This would reduce the time and cost associated with flight trials and decrease the risk to scheduling.

2. To advise pilots on adjustments to the approach path that would reduce turbulence. As an example, Figure 7 compares the approach turbulence parameter along two different approaches to the same landing spot in all wind conditions. During the FOCFTs, pilots reported lower levels of turbulence along the updated approach path (shown in red) compared to the original approach path (shown in blue). As shown in the figure, this observation is consistent with this analysis.

3. To help fill in the parts of the operating envelope that have not been tested.

4. To manage through-life risk, by assessing the impact of changes to a ship's superstructure (or on-deck equipment) on the airwake without undertaking additional trials.

5. To allow naval architects to understand the impact of design decisions on the safe operating limits of aircraft during the design of a ship.
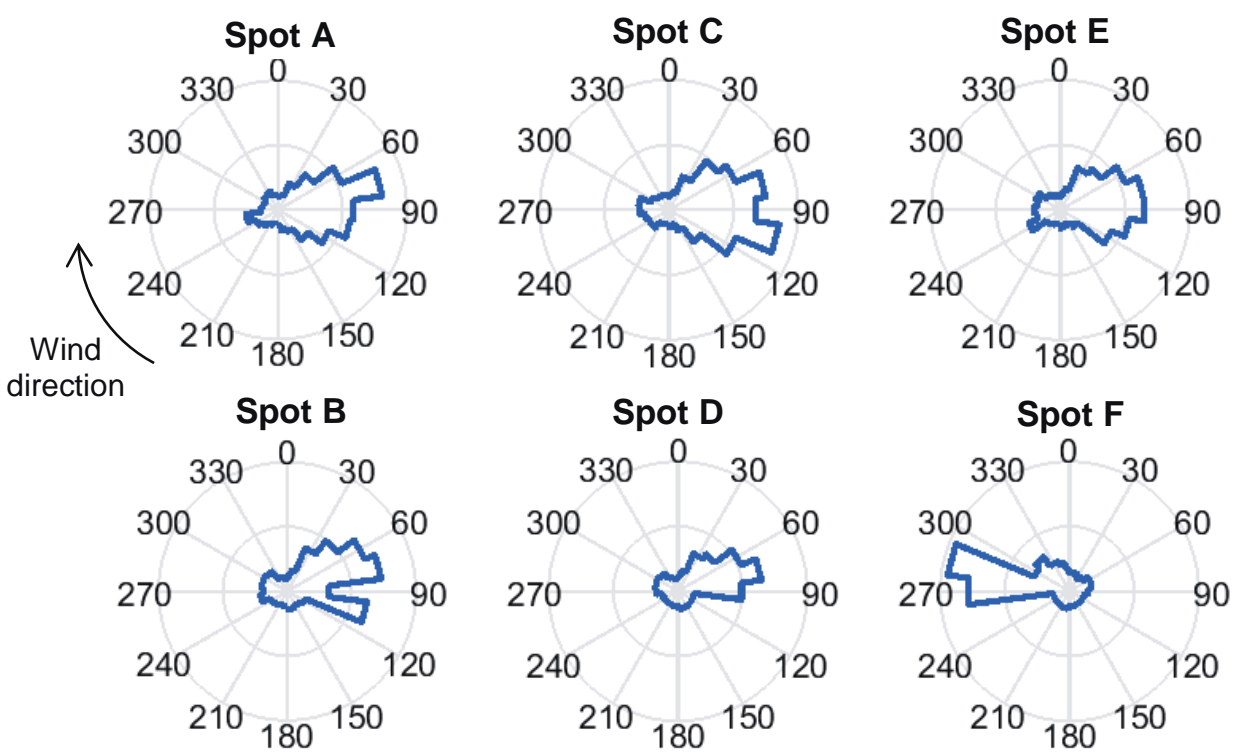

Figure 6: The variation in approach turbulence parameter with wind direction for approaches to six landing spots. 


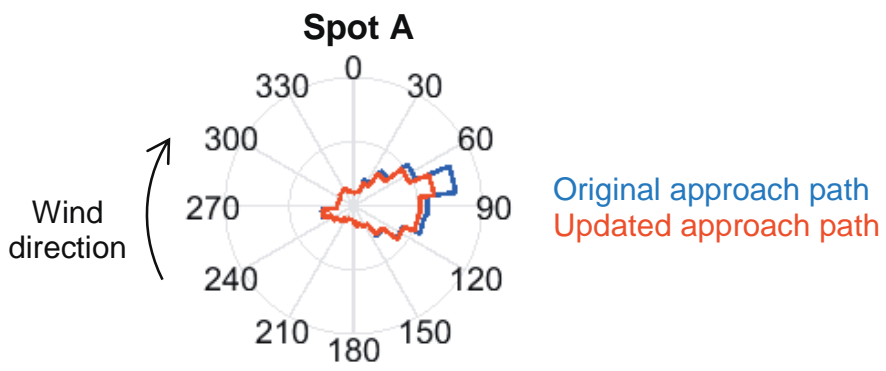

Figure 7: The variation in approach turbulence parameter with wind direction for two different approaches to the same landing spot.

\section{De-risking F-35 trials and operations}

Assessments similar to those described in Section 4 were also undertaken to de-risk the F-35 FOCFTs and operations. Typical results are provided in Figure 8, which shows contours of turbulent kinetic energy and mean vertical velocity on a plane along the SRVL approach. BAE Systems' ship-air integration team was briefed with the results of these assessments ahead of trials. The remainder of this section discusses the results of a particular assessment which suggests that it may be possible to develop a real-time system to de-risk F-35 operations and potentially expand the operating envelope.
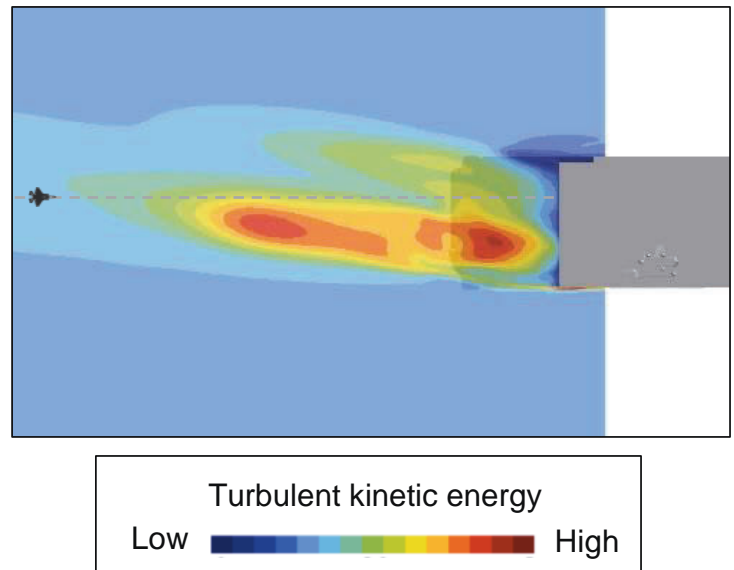

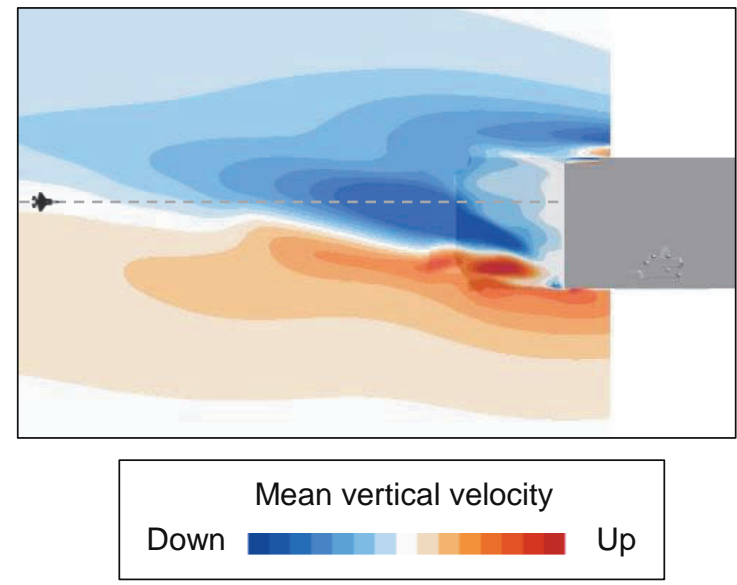

Figure 8: Contours of turbulent kinetic energy (left) and mean vertical velocity (right) on a plane along the SRVL approach path. Approximate F-35 approach shown.

Figure 9 shows a typical example of the vertical velocity fluctuations at a point on the SRVL approach as predicted by a transient CFD simulation. Initially the fluctuations are relatively small, but there is a significant increase in their magnitude after approximately 45 seconds, without any change in the nominal wind conditions. These long-term variations are also apparent in the LiDAR measurements. If it is possible to predict when these quiescent and turbulent periods will occur then this finding presents an opportunity to expand the safe operating envelope of the aircraft into wind conditions that would otherwise been deemed unsafe.

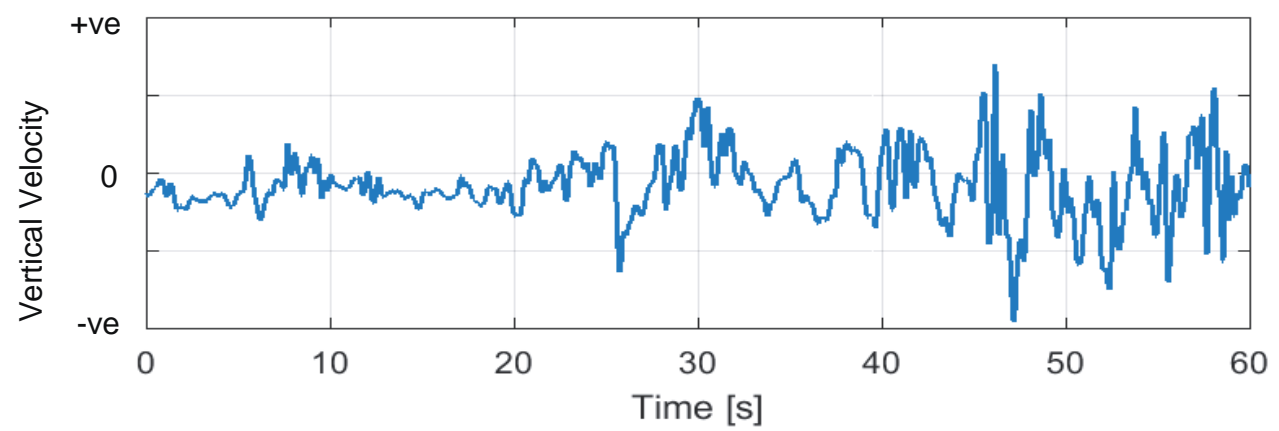

Figure 9: Typical vertical velocity fluctuations at a point in space on the SRVL approach path. 
The cause of these long-term variations is illustrated in Figure 10, which shows contours of velocity magnitude around the ship at four instances in time. Initially there is very little turbulence in the airwake, and the flow around the ship is aligned with the nominal wind direction. However, a large atmospheric disturbance can be seen upstream. When this disturbance reaches the ship, it alters the local flow direction, causing turbulence to be generated around the islands. This turbulence is then washed downstream, passing through the SRVL approach path and causing the increase in velocity fluctuations shown in Figure 9.

Based on these findings, a programme of work has been proposed to develop a real-time LiDAR measurement system that can identify upstream atmospheric disturbances and use this information to predict turbulent and quiescent periods in the airwake.
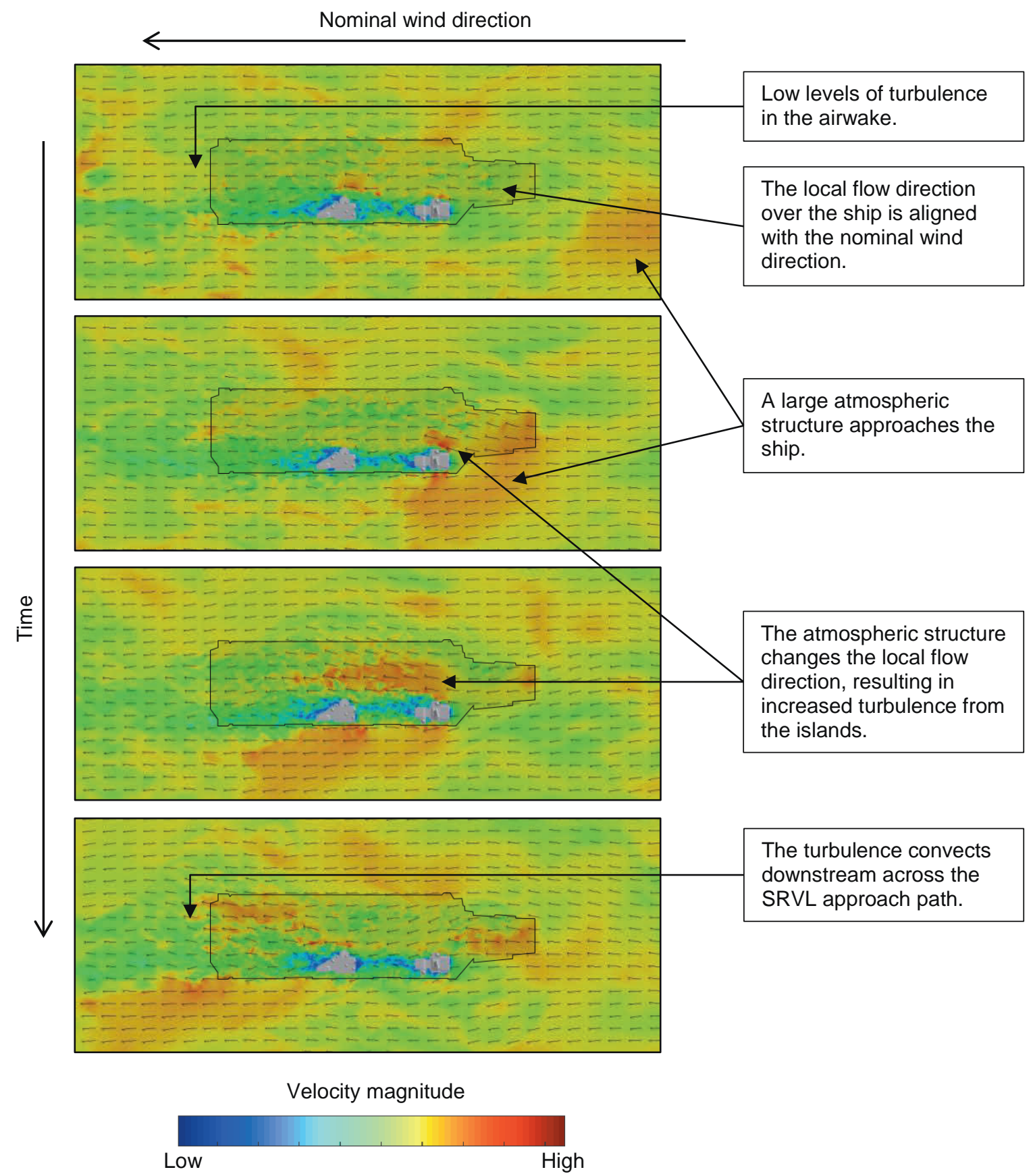

Figure 10: Contours of velocity magnitude above the flight deck showing the impact of atmospheric turbulence on the airwake. In-plane velocity vectors are shown in black. 


\section{De-risking on-deck operations}

In addition to de-risking air operations, the CFD simulations were also used to de-risk on-deck operations by ensuring that aircraft exposure limits are not exceeded. Exposure limits are provided by the aircraft manufacturer and define the maximum wind speed at which it is safe to leave aircraft tied-down. Exposure to flow speeds greater that these limits can result in failure of the tie-down lines and even damage to the aircraft, posing a significant risk to on-deck personnel and well as the aircraft and the vessel. In practice, there are two challenges around meeting these exposure limits:

1. The crew cannot measure the actual wind speed around the aircraft and, therefore, it is assumed to be equal to the wind over deck speed measured by the ship's anemometers. However, the local flow speeds around the vessel can be much greater than the nominal wind over deck speed.

2. The exposure limits assume that there is no vertical component to the flow. While this is a reasonable assumption for most airfields, it is not the case for the flow around ships (as shown in Figure 3). Ideally the exposure limits should be updated to take these vertical flows into consideration. However, as this is currently isn't the case it is desirable to avoid securing the aircraft on regions of the deck with strong vertical flows to minimise the likelihood of any failures.

To address these two issues, images such as those shown in Figure 3 were provided to the Merlin and Chinook FOCFTs teams ahead of the trials. During the trials the team used the images to identify suitable locations to secure the aircraft on-deck.

A second study was undertaken to help the Aircraft Carrier Alliance define safe operating limits when aircraft are on the lifts. To achieve this, the local flow velocities around the aircraft lifts were compared to the nominal wind over deck speed. Typical outputs from this study are provided in Figure 11, which shows contours of the ratio of peak velocity to the nominal wind over deck speed. The images also show the in-plane velocity vectors, which indicate where there are regions of vertical flow. The study found that the local flow velocities around the lifts can be up to $40 \%$ greater than the nominal wind over deck speed, although the actual increase was strongly dependent on the wind direction and lift position. As shown in Figure 11, the study also found that in some wind directions there are relatively strong vertical components to the flow over the lifts, which could pose a risk to the aircraft and should be considered in defining the safe operating limits.
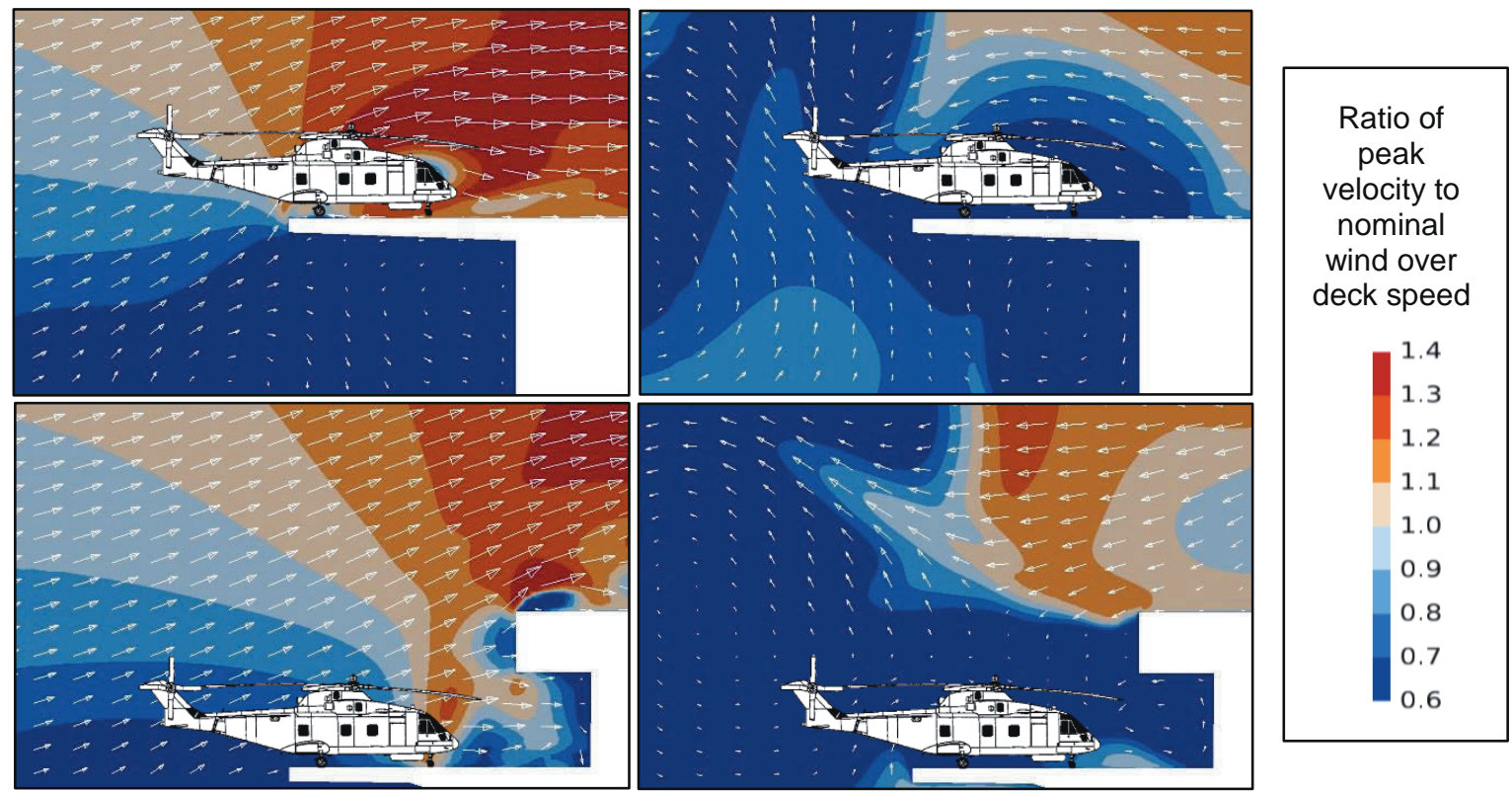

Figure 11: The ratio of peak flow velocity to the nominal wind over deck speed around the aircraft lifts in typical Red (left) and Green (right) winds, with lifts in the raised (top) and lowered (bottom) positions. In-plane flow vectors are shown in white. 


\section{Conclusions}

This paper has discussed how CFD airwake simulations have been used to de-risk flight trials and operations on the QEC carriers.

Comparing the CFD results to LiDAR and anemometer measurements has provided excellent validation of the CFD and shown that simulations could be used to calibrate the ships' anemometers to compensate for local flow effects, thereby improving the system's accuracy. This would reduce the risks associated with uncertainties in the wind speed and direction measurement during trials and operations.

To de-risk Merlin and Chinook FOCFTs, CFD simulations were used to identify the key flow features of interest to aircraft pilots. QinetiQ's FOCFTs teams and test pilots were briefed with the findings in advance of the trials to help them understand what to expect. A post trials meeting indicated that the results from the analysis correlated well with the pilots' experiences. In addition, the approach turbulence parameter was defined as a proxy measure for pilot workload. It was demonstrated that this parameter could be used to determine which wind conditions were likely to prove the most turbulent, and to identify alternative approaches to reduce pilot workload.

To de-risk F-35 operations, the simulations were used to assess the unsteady velocity fluctuations in the airwake. It was shown that the magnitude of the fluctuations can vary significantly over time for the same nominal wind conditions. These changes are due to large flow structures in the atmosphere that alter the local wind conditions around the ship. It was postulated that if these structures can be identified in the incoming wind (e.g. with a LiDAR) then it may be possible to predict the turbulent and quiescent periods of the airwake. This information could be used to expand the operating envelope of the aircraft.

The CFD simulations were also used to de-risk on-deck operations by identifying regions of strong up and down drafts around the deck that could pose a risk to aircraft. The results were used by QinetiQ's FOCFTs teams to locate suitable tie-down positions for the aircraft during the trials. The flow around the lifts was also studied to help the Aircraft Carrier Alliance ensure that aircraft are within their expose limits when on the lifts. It was shown that the local flow velocities around the lifts can be significantly higher than the nominal wind over deck speeds, and this information should be considered when determining suitable operating limits.

\section{Acknowledgements}

The author would like to thank: Michael Belmont and Jacqueline Christmas from the University of Exeter for the many discussions on the work, John Duncan from the MOD for his funding and support for the work, and James Horton for his input and advice.

\section{References}

Christmas J., 'The use of Doppler Lidar to measure ship air wakes; Results from the QEC AFAP Trial', Report No. Ex/1/18 v0.1 Draft, March 2018.

Polsky S., 'Development and Application of the SAFEDI Tool for Virtual Dynamic Interface Ship Airwake Analysis', 54th AIAA Aerospace Sciences Meeting, AIAA SciTech Forum, AIAA 2016-1771, SanDiego CA, 2016.

Pope S., 'Turbulent Flows', Cambridge University Press, June 2000. 